\title{
A MEMORY TYPE BOUNDARY STABILIZATION FOR AN EULER-BERNOULLI BEAM UNDER BOUNDARY OUTPUT FEEDBACK CONTROL
}

\author{
Yong Han Kang, Jong Yeoul Park, and Jung Ae Kim
}

\begin{abstract}
In this paper, the memory type boundary stabilization for an Euler-Bernoulli beam with one end fixed and control at the other end is considered. We prove the existence of solutions using the Galerkin method and then investigate the exponential stability of solutions by using multiplier technique.
\end{abstract}

\section{Introduction}

In this paper, we consider the following Euler-Bernoulli beam with memory which has one end fixed and control input at the other end:

$$
\begin{aligned}
& y_{t t}(x, t)+y_{x x x x}(x, t)-\int_{0}^{t} \kappa(t-\tau) y_{x x x x}(x, \tau) d \tau+g\left(y_{t}(x, t)\right)=0, \\
& \quad x \in(0, L), \quad t \geq 0, \\
& y(0, t)=y_{x}(0, t)=y_{x x}(L, t)=0, \quad t \geq 0, \\
& y_{x x x}(L, t)-\int_{0}^{t} \kappa(t-\tau) y_{x x x}(L, \tau) d \tau=u(t)-\tilde{\theta} \sin t, \quad t \geq 0, \\
& y(x, 0)=y_{0}(x), \quad y_{t}(x, 0)=y_{1}(x), \quad x \in(0, L), \\
& y_{\text {out }}(t)=y_{t}(L, t), \quad t \geq 0,
\end{aligned}
$$

where $\kappa$ represents the kernel of memory term, $g: \mathbb{R} \rightarrow \mathbb{R}$ is a given function, $\tilde{\theta}$ is a positive constant, $u: \mathbb{R}^{+} \rightarrow \mathbb{R}$ is the boundary control force applied at the free end of the beam and $y_{\text {out }}(t)$ stands for the measured signal of the system at time $t$. System (1.1) describes the transverse vibration of an extensible beam clamped at $x=0$ and supported at $x=L$ by a control force. The advantage of the adaptive stabilization is that stabilization and good control

Received December 2, 2010; Revised February 2, 2012.

2010 Mathematics Subject Classification. 49K20, 65N30, 93B52, 74G25, 74G30.

Key words and phrases. Euler-Bernoulli beam, output feedback control, memory, Galerkin method.

This work was supported by research grants from the Catholic University of Daegu in 2012 
performance can be automatically achieved even in the presence of various types of uncertainty. In this paper, we consider the stabilization of the system (1.1). To this end, we design the following adaptive output feedback controller:

$$
\begin{aligned}
& u(t)=h(t) y_{t}(L, t)+\theta(t) \sin t, \quad t \geq 0, \\
& h_{t}(t)=r y_{t}^{2}(L, t), \quad h(0)=h_{0}>0, \quad t \geq 0, \quad r>0, \\
& \theta_{t}(t)=y_{t}(L, t) \sin t, \quad \theta(0)=\theta_{0}, \quad t \geq 0,
\end{aligned}
$$

where $\theta_{0}$ is the initial condition of the estimator.

Under this adaptive controller, the closed-loop system (1.1) becomes

$$
\begin{aligned}
& y_{t t}(x, t)+y_{x x x x}(x, t)-\int_{0}^{t} \kappa(t-\tau) y_{x x x x}(x, \tau) d \tau+g\left(y_{t}(x, t)\right)=0, \\
& \quad x \in(0, L), \quad t \geq 0, \\
& y(0, t)=y_{x}(0, t)=y_{x x}(L, t)=0, \quad t \geq 0, \\
& y_{x x x}(L, t)-\int_{0}^{t} \kappa(t-\tau) y_{x x x}(L, \tau) d \tau=h(t) y_{t}(L, t)+[\theta(t)-\widetilde{\theta}] \sin t, \quad t \geq 0, \\
& y(x, 0)=y_{0}(x), \quad y_{t}(x, 0)=y_{1}(x), \quad x \in(0, L), \\
& h_{t}(t)=r y_{t}^{2}(L, t), \quad h(0)=h_{0}>0, \quad t \geq 0, \quad r>0 \\
& \theta_{t}(t)=y_{t}(L, t) \sin t, \quad \theta(0)=\theta_{0} .
\end{aligned}
$$

The energy of the system (1.3) is given by

$$
E(t)=\frac{1}{2} \int_{0}^{t}\left(y_{t}^{2}(x, t)+y_{x x}^{2}(x, t)\right) d x .
$$

Ma $[12,13]$ studied the boundary stabilization for a nonlinear beam on elastic bearings without memory and boundary output feedback control. The existence and uniform decay of solutions for an Euler-Bernoulli beam with memory was consider by Park and Kim [15]. Also, the Euler-Bernoulli beam equation with memory and boundary output feedback control was studied by Park, Kang and Kim [14]; they proved the existence and exponential stability of solutions for the following system:

$$
\begin{aligned}
& y_{t t}(x, t)+y_{x x x x}(x, t)-\int_{0}^{t} \kappa(t-\tau) y_{x x x x}(x, \tau) d \tau+g\left(y_{t}(x, t)\right)=0, \\
& \quad x \in(0, L), \quad t \geq 0 \\
& y(0, t)=y_{x}(0, t)=y_{x x}(L, t)=0, \quad t \geq 0 \\
& y_{x x x}(L, t)-\int_{0}^{t} \kappa(t-\tau) y_{x x x}(L, \tau) d \tau=h(t) y_{t}(L, t), \quad t \geq 0 \\
& y(x, 0)=y_{0}(x), \quad y_{t}(x, 0)=y_{1}(x), \quad x \in(0, L), \\
& h_{t}(t)=r y_{t}^{2}(L, t), \quad h(0)=h_{0}>0, \quad t \geq 0, \quad r>0
\end{aligned}
$$


Conrad and Omër [3] proved the existence and uniform decay for a flexible beam with a tip mass. The boundary stabilization and boundary controllability for the beams were consider by authors $[1,2,4-6,9-11,16,17]$. Guo and Luo [8] studied the stabilization and parameter estimation for a flexible-beam vibration with gain adaptive direct strain feedback control. Recently, Guo and Guo [7] consider the adaptive stabilization for a Kirchhoff-type nonlinear beam under boundary output feedback control. Motivated by [14] and some idea in [7], we can obtain our main results. The objective of this paper is to study the stabilization for a more general Euler-Bernoulli beam with memory under boundary output feedback control. Our choice of boundary feedback is motivated by the fact that boundary controls are easily implemented as the need to act only on the boundary of the spatial domain. This paper is organized as follows. In Section 2, using a constructive Galerkin approximation scheme, we show the existence and uniqueness of the solution for the system (1.3). The exponential decay that is dependent on the initial data is obtained in Section 3 by making use of the multiplier technique.

\section{Existence result}

In this section we prepare some notation and hypotheses which will be needed in the proof of our result. Let $L^{2}(0, L)$ be the usual Hilbert space with the inner product $(\cdot, \cdot)$ and the inner product induced norm $\|\cdot\|$. Throughout this paper, we define $V=\left\{y \in H^{2}(0, L) \mid y(0)=y_{x}(0)=0\right\}$ equipped with the norm $\|y\|_{V}=\left\|y_{x x}\right\|, W=\left\{y \in V \cap H^{4}(0, L) \mid y_{x x}(L)=0\right\}$ equipped with the norm $\|y\|_{W}=\left\|y_{x x}\right\|+\left\|y_{x x x x}\right\|$ and $(u, v)=\int_{0}^{L} u(x) v(x) d x$. From the Poincaré inequality, it follows that $\|\cdot\|_{V}$ and $\|\cdot\|_{W}$ are equivalent to the standard norms of $H^{2}(0, L)$ and $H^{4}(0, L)$, respectively. Now, we state the following hypotheses which will be assumed in this paper.

$\left(H_{1}\right)$ For any $y_{0} \in W, y_{1} \in V$,

$$
\begin{aligned}
& y_{x x x x}(x, 0)+g\left(y_{1}\right)=0, \quad x \in(0, L), \\
& y(0,0)=y_{x}(0,0)=y_{x x}(L, 0)=0, \\
& y_{x x x}(L, 0)=h_{0} y_{t}(L, 0) .
\end{aligned}
$$

$\left(H_{2}\right)$ Let $g: \mathbb{R} \rightarrow \mathbb{R}$ be a continuously differentiable function and there exists a positive constant $\mu$ such that

$$
g(0)=0, \quad(g(r)-g(s))(r-s) \geq \mu|r-s|^{2}, \quad r, s \in \mathbb{R} .
$$

$\left(H_{3}\right)$ Let the function $\kappa: \mathbb{R}^{+} \rightarrow \mathbb{R}^{+}$be a positive and bounded $C^{2}$-function such that

$$
\ell=1-\int_{0}^{\infty} \kappa(r) d r>0
$$

and for some positive $m_{i}, i=0,1,2$

$$
-m_{0} \kappa(t) \leq \kappa_{t}(t) \leq-m_{1} \kappa(t), \quad \forall t \geq 0,
$$




$$
0 \leq \kappa_{t t}(t) \leq m_{2} \kappa(t), \quad \forall t \geq 0 .
$$

Considering the above hypotheses we have the following existence result by Galerkin method.

Theorem 2.1. Let $y_{0} \in W, y_{1} \in V$. Suppose that $\left(H_{1}\right),\left(H_{2}\right)$ and $\left(H_{3}\right)$ are satisfied. Then the problem (1.3) has a unique solution $y$ in the sense that for any time $T>0$,

$$
\begin{aligned}
& y \in L^{\infty}(0, T ; W), \quad y_{t} \in L^{\infty}(0, T ; V), \quad y_{t t} \in L^{\infty}\left(0, T ; L^{2}(0, L)\right), \\
& h \in C^{1}[0, T], \quad \theta \in C^{1}[0, T] \\
& y_{t t}(x, t)+y_{x x x x}(x, t)-\int_{0}^{t} \kappa(t-\tau) y_{x x x x}(x, \tau) d \tau+g\left(y_{t}(x, t)\right)=0 \\
& i n \quad L^{2}\left(0, T ; L^{2}(0, L)\right), \\
& y(0, t)=y_{x}(0, t)=y_{x x}(L, t)=0, \quad t \geq 0, \\
& y_{x x x}(L, t)-\int_{0}^{t} \kappa(t-\tau) y_{x x x}(L, t) d x=h(t) y_{t}(L, t)+[\theta(t)-\widetilde{\theta}] \sin t, \quad t \geq 0, \\
& y(x, 0)=y_{0}(x), \quad y_{t}(x, t)=y_{1}(x), \quad x \in(0, L), \\
& h_{t}(t)=r y_{t}^{2}(L, t), \quad t \geq 0, \quad h(0)=h_{0}>0, \quad r>0 \\
& \theta_{t}(t)=y_{t}(L, t) \sin t, \quad t \geq 0, \quad \theta(0)=\theta_{0} .
\end{aligned}
$$

By the Sobolev embedding theorem, it follows that $y \in C((0, L) \times[0, T])$.

Proof. Let us solve the variational problem with (2.5) which is given by: find $y(t) \in V$ such that

$$
\begin{aligned}
& \left(y_{t t}(t), w\right)+\left(y_{x x}(t), w_{x x}\right)-\int_{0}^{t} \kappa(t-\tau)\left(y_{x x}(\tau), w_{x x}\right) d \tau+\left(g\left(y_{t}(t)\right), w\right) \\
& +\left\{h(t) y_{t}(L, t)+(\theta(t)-\widetilde{\theta}) \sin t\right\} w(L)=0 \text { for all } w \in V .
\end{aligned}
$$

Let $\left\{w^{j}\right\}$ be a complete orthogonal system of $V$ for which $\left\{y_{0}, y_{1}\right\} \in \operatorname{Span}\left\{w^{1}\right.$, $\left.w^{2}\right\}$. For each $m \in \mathbb{N}$, we denote $V_{m}=\operatorname{Span}\left\{w^{1}, w^{2}, \ldots, w^{m}\right\}$. We search for a function

$$
y^{m}(t)=\sum_{j=1}^{m} k^{j}(t) w^{j}
$$

such that for any $w \in V_{m}$, it satisfies the approximate equation

$$
\begin{aligned}
& \left(y_{t t}^{m}(t), w\right)+\left(y_{x x}^{m}(t), w_{x x}\right)-\int_{0}^{t} \kappa(t-\tau)\left(y_{x x}^{m}(\tau), w_{x x}\right) d \tau+\left(g\left(y_{t}^{m}(t)\right), w\right) \\
& \quad+\left\{h^{m}(t) y_{t}^{m}(L, t)+\left(\theta^{m}(t)-\widetilde{\theta}\right) \sin t\right\} w(L)=0, \\
& h_{t}^{m}(t)=r\left[\sum_{j=1}^{m} k_{t}^{j}(t) w^{j}(L)\right]^{2}=r\left[y_{t}^{m}(L, t)\right]^{2},
\end{aligned}
$$




$$
\begin{aligned}
& \theta_{t}^{m}(t)=\left[\sum_{j=1}^{m} k_{t}^{j}(t) w^{j}(L)\right] \sin t=y_{t}^{m}(L, t) \sin t, \\
& h^{m}(0)=h_{0}>0, \quad \theta^{m}(0)=\theta_{0}, \\
& y^{m}(0)=y_{0 m} \rightarrow y_{0} \text { in } W, \quad y_{t}^{m}(0)=y_{1 m} \rightarrow y_{1} \text { in } V .
\end{aligned}
$$

By standard methods in differential equations, we can prove the existence of a solution to $(2.7)$ on some interval, $\left(0, t_{m}\right)$, where $t_{m}=\infty$ by using the first estimate below. In order to prove Theorem 2.1 it suffices to prove the following a priori estimates.

Estimate I. Replacing $w$ by $y_{t}^{m}$ in (2.7), we have

$$
\begin{aligned}
& \frac{1}{2} \frac{d}{d t}\left(\left\|y_{t}^{m}(t)\right\|^{2}+\left\|y_{x x}^{m}(t)\right\|^{2}\right)+\left(g\left(y_{t}^{m}(t)\right), y_{t}^{m}(t)\right) \\
= & \frac{d}{d t}\left(\int_{0}^{t} \kappa(t-\tau)\left(y_{x x}^{m}(\tau), y_{x x}^{m}(t)\right) d \tau\right)-\int_{0}^{t} \kappa_{t}(t-\tau)\left(y_{x x}^{m}(\tau), y_{x x}^{m}(t)\right) d \tau \\
& -\kappa(0)\left\|y_{x x}^{m}(t)\right\|^{2}-\left\{h^{m}(t)\left[y_{t}^{m}(L, t)\right]^{2}+\left[\theta^{m}(t)-\tilde{\theta}\right] \sin t \cdot y_{t}^{m}(L, t)\right\} .
\end{aligned}
$$

By hypotheses (2.4) and Cauchy-Schwartz inequality, we deduce that

$$
\begin{aligned}
& \left|\int_{0}^{t} \kappa_{t}(t-\tau)\left(y_{x x}^{m}(\tau), y_{x x}^{m}(t)\right) d \tau\right| \\
\leq & \left\|y_{x x}^{m}(t)\right\| \int_{0}^{t}\left|\kappa_{t}(t-\tau)\right|\left\|y_{x x}^{m}(\tau)\right\| d \tau \\
\leq & \frac{m_{0}^{2}}{2}\left\|y_{x x}^{m}(t)\right\|^{2}+\frac{1}{2}\left(\int_{0}^{t} \kappa(t-\tau)\left\|y_{x x}^{m}(\tau)\right\| d \tau\right)^{2} \\
\leq & \frac{m_{0}^{2}}{2}\left\|y_{x x}^{m}(t)\right\|^{2}+\frac{1}{2}\|\kappa\|_{L^{1}(0, \infty)} \int_{0}^{t} \kappa(t-\tau)\left\|y_{x x}^{m}(\tau)\right\|^{2} d \tau .
\end{aligned}
$$

Integrating (2.8) over $(0, t)$ and using $(2.2)$ and (2.9), we obtain

$$
\begin{aligned}
& \left\|y_{t}^{m}(t)\right\|^{2}+\left\|y_{x x}^{m}(t)\right\|^{2}+\frac{1}{r}\left[h^{m}(t)\right]^{2}+\left[\theta^{m}(t)-\widetilde{\theta}\right]^{2}+2 \mu \int_{0}^{t}\left\|y_{t}^{m}(\tau)\right\|^{2} d \tau \\
& \leq\left\|y_{t}^{m}(0)\right\|^{2}+\left\|y_{x x}^{m}(0)\right\|^{2}+\frac{1}{r}\left[h^{m}(0)\right]^{2}+\left[\theta_{0}-\widetilde{\theta}\right]^{2} \\
& +2 \int_{0}^{t} \kappa(t-\tau)\left(y_{x x}^{m}(\tau), y_{x x}^{m}(t)\right) d \tau \\
& +\left(m_{0}^{2}+\|\kappa\|_{L^{1}(0, \infty)}^{2}-2 \kappa(0)\right) \int_{0}^{t}\left\|y_{x x}(\tau)\right\|^{2} d \tau
\end{aligned}
$$

Using Schwartz inequality and Young's inequality, we get

$$
\begin{aligned}
& \left|\int_{0}^{t} \kappa(t-\tau)\left(y_{x x}^{m}(\tau), y_{x x}^{m}(t)\right) d \tau\right| \\
\leq & \left\|y_{x x}^{m}(t)\right\|\|\kappa\|_{L^{1}(0, \infty)}^{\frac{1}{2}}\left(\int_{0}^{t} \kappa(t-\tau)\left\|y_{x x}^{m}(\tau)\right\|^{2} d \tau\right)^{\frac{1}{2}}
\end{aligned}
$$




$$
\leq \frac{1}{8}\left\|y_{x x}^{m}(t)\right\|^{2}+2\|\kappa\|_{L^{1}(0, \infty)}\|\kappa\|_{L^{\infty}(0, \infty)} \int_{0}^{t}\left\|y_{x x}^{m}(\tau)\right\|^{2} d \tau
$$

Combining the inequalities (2.10), (2.11) and applying Gronwall's Lemma, we see the first estimate:

$$
\left\|y_{t}^{m}(t)\right\|^{2}+\left\|y_{x x}^{m}(t)\right\|^{2}+\frac{1}{r}\left[h^{m}(t)\right]^{2}+\left[\theta^{m}(t)-\widetilde{\theta}\right]^{2} \leq M_{1},
$$

where $M_{1}>0$ depends on the initial data $y_{0}, y_{1}, h_{0}, \theta_{0}$.

From this, we obtain $y_{t}^{m}(L, t) \in L^{\infty}(0, \infty)$. Therefore, the approximate solution can be extended to the whole interval $[0, T]$, where $T=\infty$.

Estimate II. First of all, we estimate the $L^{2}$-norm of $y_{t t}^{m}(0)$. Considering $t=0$ and $w=y_{t t}^{m}(0)$ in $(2.7)$, then we get

$$
\left\|y_{t t}^{m}(0)\right\|^{2}+\left(y_{x x}^{m}(0), y_{x x t t}^{m}(0)\right)+\left(g\left(y_{t}^{m}(0)\right), y_{t t}^{m}(0)\right)+h_{0} y_{t}^{m}(L, 0) y_{t t}^{m}(L, 0)=0 .
$$

Since the compatibility condition $\left(H_{1}\right)$, we obtain

$$
\begin{aligned}
\left(y_{x x}^{m}(0), y_{x x t t}^{m}(0)\right)= & y_{x x}^{m}(L, 0) y_{x t t}^{m}(L, 0)-y_{x x}^{m}(0,0) y_{x t t}^{m}(0,0)-y_{x x x}^{m}(L, 0) y_{t t}^{m}(L, 0) \\
& +y_{t t}^{m}(0,0) y_{x x x}^{m}(0,0)+\left(y_{x x x x}^{m}(0), y_{t t}^{m}(0)\right) \\
= & -h_{0} y_{t}^{m}(L, 0) y_{t t}^{m}(L, 0)-\left(g\left(y_{t}^{m}(0)\right), y_{t t}^{m}(0)\right) .
\end{aligned}
$$

Therefore, from the above equality, there exists the following result

$$
\left\|y_{t t}^{m}(0)\right\|=0 \text {. }
$$

Finally, differentiating (2.7) and writing the equation with $w=y_{t t}^{m}(t)$, we have

$$
\begin{aligned}
& \frac{1}{2} \frac{d}{d t}\left(\left\|y_{t t}^{m}(t)\right\|^{2}+\left\|y_{x x t}^{m}(t)\right\|^{2}\right)+\kappa(0)\left\|y_{x x t}^{m}(t)\right\|^{2}+\left(g^{\prime}\left(y_{t}^{m}(t)\right) y_{t t}^{m}(t), y_{t t}^{m}(t)\right) \\
& +\left(r\left[y_{t}^{m}(L, t)\right]^{3}+h^{m}(t) y_{t t}^{m}(L, t)\right) y_{t t}^{m}(L, t) \\
& +\left(y_{t}^{m}(L, t) \sin ^{2} t+\left(\theta^{m}(t)-\widetilde{\theta}\right) \cos t\right) y_{t t}^{m}(L, t) \\
= & \frac{d}{d t}\left(\int_{0}^{t} \kappa_{t}(t-\tau)\left(y_{x x}^{m}(\tau), y_{x x t}^{m}(t)\right) d \tau\right)-\int_{0}^{t} \kappa_{t t}(t-\tau)\left(y_{x x}^{m}(\tau), y_{x x t}^{m}(t)\right) d \tau \\
& -\kappa_{t}(0)\left(y_{x x}^{m}(t), y_{x x t}^{m}(t)\right)+\kappa(0) \frac{d}{d t}\left(y_{x x}^{m}(t), y_{x x t}^{m}(t)\right) .
\end{aligned}
$$

From hypotheses (2.4), we deduce that

$$
\begin{aligned}
& \left|\int_{0}^{t} \kappa_{t t}(t-\tau)\left(y_{x x}^{m}(\tau), y_{x x t}^{m}(t)\right) d \tau\right| \\
\leq & \frac{m_{2}^{2}}{2}\left\|y_{x x t}(t)\right\|^{2}+\frac{1}{2}\|\kappa\|_{L^{1}(0, \infty)} \int_{0}^{t} \kappa(t-\tau)\left\|y_{x x}^{m}(\tau)\right\|^{2} d \tau .
\end{aligned}
$$

Since $g \in C^{1}(\mathbb{R})$ and $y_{t}^{m}(t)$ is bounded by (2.12), there exists $C_{1}>0$ depends on the initial datas $y_{0}, y_{1}, h_{0}, \theta_{0}$ such that

$$
\left|\left(g^{\prime}\left(y_{t}^{m}(t)\right) y_{t t}^{m}(t), y_{t t}^{m}(t)\right)\right| \leq C_{1}\left\|y_{t t}^{m}(t)\right\|^{2} .
$$


Using $(2.13),(2.15)$ and $(2.16)$, we integrate $(2.14)$ over $(0, t)$ to obtain

$$
\begin{aligned}
& \frac{1}{2}\left\|y_{t t}^{m}(t)\right\|^{2}+\frac{1}{2}\left\|y_{x x t}^{m}(t)\right\|^{2}+\kappa(0) \int_{0}^{t}\left\|y_{x x t}^{m}(\tau)\right\|^{2} d \tau+\frac{r}{4}\left[y_{t}^{m}(L, t)\right]^{4} \\
\leq & C_{2}+\frac{m_{2}^{2}}{2} \int_{0}^{t}\left\|y_{x x t}^{m}(\tau)\right\|^{2} d \tau+\frac{1}{2}\|\kappa\|_{L^{1}(0, \infty)}^{2} \int_{0}^{t}\left\|y_{x x}^{m}(\tau)\right\|^{2} d \tau \\
& +C_{1} \int_{0}^{t}\left\|y_{t t}^{m}(\tau)\right\|^{2} d \tau+\kappa(0)\left(y_{x x}^{m}(t), y_{x x t}^{m}(t)\right)-\kappa_{t}(0) \int_{0}^{t}\left(y_{x x}^{m}(\tau), y_{x x t}^{m}(\tau)\right) d \tau \\
& +\int_{0}^{t} \kappa_{t}(t-\tau)\left(y_{x x}^{m}(\tau), y_{x x t}^{m}(t)\right) d \tau+\frac{r}{4}\left[y_{t}^{m}(L, 0)\right]^{4}-\int_{0}^{t} h^{m}(\tau)\left[y_{t t}^{m}(L, \tau)\right]^{2} d \tau \\
& -\frac{1}{2}\left[y_{t}^{m}(L, t)\right]^{2} \sin ^{2} t+\frac{1}{2} \int_{0}^{t}\left[y_{t}^{m}(L, \tau)\right]^{2} \sin 2 \tau d \tau-y_{t}^{m}(L, t)\left[\theta^{m}(t)-\widetilde{\theta}\right] \cos t \\
& +y_{t}^{m}(L, 0)\left[\theta_{0}-\widetilde{\theta}\right]+\int_{0}^{t} y_{t}^{m}(L, \tau)\left\{y_{t}^{m}(L, \tau) \sin \tau \cos \tau-\left[\theta^{m}(\tau)-\widetilde{\theta}\right] \sin \tau\right\} d \tau,
\end{aligned}
$$

where $C_{2}>0$ depends on the initial data $y_{0}, y_{1}, h_{0}$.

Now, since (2.4), for any $\eta>0$ we see that

$$
\left|\kappa(0)\left(y_{x x}^{m}(t), y_{x x t}^{m}(t)\right)\right| \leq \frac{(\kappa(0))^{2}}{4 \eta}\left\|y_{x x}^{m}(t)\right\|^{2}+\eta\left\|y_{x x t}^{m}(t)\right\|^{2},
$$

$\left|\kappa_{t}(0) \int_{0}^{t}\left(y_{x x}^{m}(\tau), y_{x x t}^{m}(\tau)\right) d \tau\right| \leq \frac{m_{0}^{2}(\kappa(0))^{2}}{4 \eta} \int_{0}^{t}\left\|y_{x x}^{m}(\tau)\right\|^{2} d \tau+\eta \int_{0}^{t}\left\|y_{x x t}^{m}(\tau)\right\|^{2} d \tau$ and

$$
\begin{aligned}
& \left|\int_{0}^{t} \kappa_{t}(t-\tau)\left(y_{x x}^{m}(\tau), y_{x x t}^{m}(t)\right) d \tau\right| \\
\leq & \frac{m_{0}^{2}}{4 \eta}\|\kappa\|_{L^{1}(0, \infty)}\|\kappa\|_{L^{\infty}(0, \infty)} \int_{0}^{t}\left\|y_{x x}^{m}(\tau)\right\|^{2} d \tau+\eta\left\|y_{x x t}^{m}(t)\right\|^{2} .
\end{aligned}
$$

From the inequality $a b \leq \delta_{1} a^{2}+\frac{1}{4 \delta_{1}} b^{2}, \delta_{1}>0$ and the Sobolev embedding theorem $\left\|y_{t}(L, t)\right\|^{2} \leq \delta_{2}\left\|y_{x x t}(t)\right\|^{2}, \delta_{2}>0$, we deduce

$$
\begin{aligned}
& \left|-\frac{1}{2}\left[y_{t}^{m}(L, t)\right]^{2} \sin ^{2} t-y_{t}^{m}(L, t)\left[\theta^{m}(t)-\widetilde{\theta}\right] \cos t\right| \\
\leq & \delta_{1}\left[y_{t}^{m}(L, t)\right]^{2} \sin ^{2} t+\delta_{1}\left[y_{t}^{m}(L, t)\right]^{2} \cos ^{2} t+\frac{1}{4 \delta_{1}}\left[\theta^{m}(t)-\widetilde{\theta}\right]^{2} \\
\leq & \widetilde{\delta_{1}}\left\|y_{x x t}^{m}(t)\right\|^{2}+\frac{1}{4 \delta_{1}}\left[\theta^{m}(t)-\widetilde{\theta}\right]^{2}, \text { where } \widetilde{\delta_{1}}=\delta_{1} \delta_{2}
\end{aligned}
$$

and using Young's inequality we obtain

$$
\left|\int_{0}^{t} y_{t}^{m}(L, \tau)\left\{y_{t}^{m}(L, \tau) \sin \tau \cos \tau-\left[\theta^{m}(\tau)-\widetilde{\theta}\right] \sin \tau\right\} d \tau\right|
$$




$$
\begin{aligned}
& \leq \int_{0}^{t}\left[y_{t}^{m}(L, \tau)\right]^{2} d \tau+\frac{1}{2} \int_{0}^{t}\left[\theta^{m}(\tau)-\widetilde{\theta}\right]^{2} d \tau \\
& \leq \frac{h^{m}(t)}{r}-\frac{h_{0}}{r}+\frac{1}{2} \int_{0}^{t}\left[\theta^{m}(\tau)-\widetilde{\theta}\right]^{2} d \tau .
\end{aligned}
$$

Thus from (2.17)-(2.22), we have

$$
\begin{aligned}
& \frac{1}{2}\left\|y_{t t}^{m}(t)\right\|^{2}+\frac{1}{2}\left\|y_{x x t}^{m}(t)\right\|^{2}+\kappa(0) \int_{0}^{t}\left\|y_{x x t}^{m}(\tau)\right\|^{2} d \tau+\frac{r}{4}\left[y_{t}^{m}(L, t)\right]^{4} \\
& \leq C_{2}+C_{1} \int_{0}^{t}\left\|y_{t t}^{m}(\tau)\right\|^{2} d \tau+\frac{(\kappa(0))^{2}}{4 \eta}\left\|y_{x x}^{m}(t)\right\|^{2} \\
& +\left(\frac{m_{2}^{2}}{2}+\eta\right) \int_{0}^{t}\left\|y_{x x t}^{m}(\tau)\right\|^{2} d \tau+2 \eta\left\|y_{x x t}^{m}(t)\right\|^{2} \\
& +\left(\frac{1}{2}\|\kappa\|_{L^{1}(0, \infty)}^{2}+\frac{m_{0}^{2}(\kappa(0))^{2}}{4 \eta}+\frac{m_{0}^{2}}{4 \eta}\|\kappa\|_{L^{1}(0, \infty)}\|\kappa\|_{L^{\infty}(0, \infty)}\right) \int_{0}^{t}\left\|y_{x x}^{m}(\tau)\right\|^{2} d \tau \\
& +\frac{r}{4}\left[y_{t}^{m}(L, 0)\right]^{4}-\int_{0}^{t} h^{m}(\tau)\left[y_{t t}^{m}(L, \tau)\right]^{2} d \tau-\frac{1}{2}\left[y_{t}^{m}(L, t)\right]^{2} \sin ^{2} t \\
& +\frac{1}{2} \int_{0}^{t}\left[y_{t}^{m}(L, \tau)\right]^{2} \sin 2 \tau d \tau-y_{t}^{m}(L, t)\left[\theta^{m}(t)-\widetilde{\theta}\right] \cos t+y_{t}^{m}(L, 0)\left[\theta_{0}-\widetilde{\theta}\right] \\
& +\int_{0}^{t} y_{t}^{m}(L, \tau)\left\{y_{t}^{m}(L, \tau) \sin \tau \cos \tau-\left[\theta^{m}(\tau)-\widetilde{\theta}\right] \sin \tau\right\} d \tau \\
& \leq C_{2}+C_{1} \int_{0}^{t}\left\|y_{t t}^{m}(\tau)\right\|^{2} d \tau+\frac{(\kappa(0))^{2}}{4 \eta}\left\|y_{x x}^{m}(t)\right\|^{2} \\
& +\left(\frac{m_{2}^{2}}{2}+\eta\right) \int_{0}^{t}\left\|y_{x x t}^{m}(\tau)\right\|^{2} d \tau+2 \eta\left\|y_{x x t}^{m}(t)\right\|^{2} \\
& +\left(\frac{1}{2}\|\kappa\|_{L^{1}(0, \infty)}^{2}+\frac{m_{0}^{2}(\kappa(0))^{2}}{4 \eta}+\frac{m_{0}^{2}}{4 \eta}\|\kappa\|_{L^{1}(0, \infty)}\|\kappa\|_{L^{\infty}(0, \infty)}\right) \int_{0}^{t}\left\|y_{x x}^{m}(\tau)\right\|^{2} d \tau \\
& +\frac{r}{4}\left[y_{t}^{m}(L, 0)\right]^{4}+\frac{3 h^{m}(t)}{2 r}+\widetilde{\delta_{1}}\left\|y_{x x t}^{m}(t)\right\|^{2}+\frac{1}{4 \delta_{1}}\left[\theta^{m}(t)-\widetilde{\theta}\right]^{2}+\frac{1}{2}\left[y_{t}^{m}(L, 0)\right]^{2} \\
& +\frac{1}{2}\left[\theta_{0}-\widetilde{\theta}\right]^{2}+\frac{1}{2} \int_{0}^{t}\left[\theta^{m}(\tau)-\widetilde{\theta}\right]^{2} d \tau-\frac{3 h_{0}}{2 r} .
\end{aligned}
$$

From the inequalities (2.12) and (2.23) and choosing $\eta>0$ sufficiently small, we get

$$
\begin{aligned}
& \frac{1}{2}\left\|y_{t t}^{m}(t)\right\|^{2}+\left(\frac{1}{2}-\widetilde{\delta_{1}}\right)\left\|y_{x x t}^{m}(t)\right\|^{2}+\kappa(0) \int_{0}^{t}\left\|y_{x x t}^{m}(\tau)\right\|^{2} d \tau+\frac{r}{4}\left[y_{t}^{m}(L, t)\right]^{4} \\
\leq & C_{3}+C_{1} \int_{0}^{t}\left\|y_{t t}^{m}(\tau)\right\|^{2} d \tau+\left(\frac{m_{2}^{2}}{2}+\eta\right) \int_{0}^{t}\left\|y_{x x t}^{m}(\tau)\right\|^{2} d \tau,
\end{aligned}
$$

where $C_{3}>0$ depends on the initial data $y_{0}, y_{1}, h_{0}, \theta_{0}$. 
Taking $\widetilde{\delta_{1}}$ with $\frac{1}{2}-\widetilde{\delta_{1}}>0$ and using of Gronwall's Lemma, we have the second estimate:

$$
\left\|y_{t t}^{m}(t)\right\|^{2}+\left\|y_{x x t}^{m}(t)\right\|^{2}+r\left[y_{t}^{m}(L, t)\right]^{4} \leq M_{2},
$$

where $M_{2}$ depends on the initial data $y_{0}, y_{1}, h_{0}, \theta_{0}$.

Analysis of the nonlinear terms. By estimates (2.12) and (2.24), we deduce that

$$
\begin{aligned}
& \left\{y^{m}\right\} \text { is bounded in } L^{\infty}(0, T ; V), \\
& \left\{y_{t}^{m}\right\} \text { is bounded in } L^{\infty}(0, T ; V), \\
& \left\{y_{t}^{m}\right\} \text { is bounded in } L^{\infty}\left(0, T ; L^{2}(0, L)\right), \\
& \left\{y_{t}^{m}(L, t)\right\} \text { is bounded in } L^{2}(0, T), \\
& \left\{h^{m}\right\} \text { is bounded in } L^{\infty}(0, T), \\
& \left\{h_{t}^{m}\right\}=\left\{r\left(y_{t}^{m}(L, t)\right)^{2}\right\} \text { is bounded in } L^{\infty}(0, T), \\
& \left\{\theta^{m}\right\} \text { is bounded in } L^{\infty}(0, T), \\
& \left\{\theta_{t}^{m}\right\}=\left\{y_{t}^{m}(L, t) \sin t\right\} \text { is bounded in } L^{\infty}(0, T) .
\end{aligned}
$$

Therefore, there exists a subsequence of $\left\{y^{m}\right\}$, still denoted by $\left\{y^{m}\right\}$ such that

$$
\begin{aligned}
& y^{m} \rightarrow y \text { weakly star in } L^{\infty}(0, T ; V), \\
& y_{t}^{m} \rightarrow y_{t} \text { weakly star in } L^{\infty}(0, T ; V), \\
& y_{t t}^{m} \rightarrow y_{t t} \quad \text { weakly star in } L^{\infty}\left(0, T ; L^{2}(0, L)\right), \\
& y_{t}^{m}(L, t) \rightarrow y_{t}(L, t) \text { weakly in } L^{2}(0, T), \\
& h^{m} \rightarrow h \text { weakly star in } L^{\infty}(0, T), \\
& h_{t}^{m} \rightarrow h_{t} \text { weakly star in } L^{\infty}(0, T), \\
& \theta^{m} \rightarrow \theta \text { weakly star in } L^{\infty}(0, T), \\
& \theta_{t}^{m} \rightarrow \theta_{t} \text { weakly star in } L^{\infty}(0, T) .
\end{aligned}
$$

Due to the compact embedding $V \hookrightarrow L^{2}(0, L)$, we can get a subsequence such that

$$
y_{t}^{m} \rightarrow y_{t} \text { strongly in } L^{2}\left(0, T ; L^{2}(0, L)\right) .
$$

From $\left(H_{2}\right)$ and $(2.27)$, we get

$$
g\left(y_{t}^{m}\right) \rightarrow g\left(y_{t}\right) \text { a.e. in } x \in(0, L), \quad t>0 .
$$

From the above convergence and due to the boundedness of the sequence $\left\{g\left(y_{t}^{m}\right)\right\}$ in $L^{2}\left(0, T ; L^{2}(0, L)\right)$, we conclude by Lion's Lemma that

$$
g\left(y_{t}^{m}\right) \rightarrow g\left(y_{t}\right) \text { weakly in } L^{2}\left(0, T ; L^{2}(0, L)\right) .
$$

Moreover, by the Sobolev embedding theorem and (2.26), we see that $h \in$ $C^{1}[0, T]$ and $h^{m}(t) y_{t}^{m}(L, t) \rightarrow h(t) y_{t}(L, t)$ weakly in $L^{2}(0, T)$.

We also derive that

$$
\theta \in C^{1}[0, T] \text { and } \theta^{m}(t) \sin t \rightarrow \theta(t) \sin t \text { weakly in } L^{2}(0, T) .
$$


The above convergence results are sufficient to pass to the limit in the nonlinear terms of (2.7). Then it is a matter of routine to deduce the existence of global solutions in $[0, T]$. The uniqueness can be proved by the straightforward methods and Gronwall's inequality.

\section{Exponential stability}

Having established global existence of solution to (1.3), we focus our attention on exponential decay that can be obtained for the energy function.

We define the energy $E(t)$ of problem (1.3) by $E(t)=\frac{1}{2}\left\|y_{t}(t)\right\|^{2}+\frac{1}{2}\left\|y_{x x}(t)\right\|^{2}$. Then the derivative of the energy is given by

$$
\begin{aligned}
E_{t}(t)= & -\left(g\left(y_{t}(t)\right), y_{t}(t)\right)+\int_{0}^{t} \kappa(t-\tau)\left(y_{x x}(\tau), y_{x x t}(\tau)\right) d \tau-h(t)\left(y_{t}(L, t)\right)^{2} \\
& -[\theta(t)-\widetilde{\theta}] \sin t \cdot y_{t}(L, t) .
\end{aligned}
$$

Defining

$$
\left(\kappa \square y_{x x}\right)(t)=\int_{0}^{t} \kappa(t-\tau)\left\|y_{x x}(\tau)-y_{x x}(t)\right\|^{2} d \tau .
$$

By simple computation, we obtain

$$
\begin{aligned}
\left(\kappa \square y_{x x}\right)_{t}(t)= & \left(\kappa_{t} \square y_{x x}\right)(t)-2 \int_{0}^{t} \kappa(t-\tau)\left(y_{x x}(\tau), y_{x x t}(t)\right) d \tau \\
& +\frac{d}{d t}\left(\left\|y_{x x}(t)\right\|^{2} \int_{0}^{t} \kappa(\tau) d \tau\right)-\kappa(t)\left\|y_{x x}(t)\right\|^{2} .
\end{aligned}
$$

From (3.2), we see that

$$
\begin{aligned}
& \int_{0}^{t} \kappa(t-\tau)\left(y_{x x}(\tau), y_{x x t}(t)\right) d \tau \\
= & -\frac{1}{2}\left(\kappa \square y_{x x}\right)_{t}(t)+\frac{1}{2}\left(\kappa_{t} \square y_{x x}\right)(t)+\frac{1}{2} \frac{d}{d t}\left(\left\|y_{x x}(t)\right\|^{2} \int_{0}^{t} \kappa(\tau) d \tau\right) \\
& -\frac{1}{2} \kappa(t)\left\|y_{x x}(t)\right\|^{2} .
\end{aligned}
$$

Define the modified energy by

$$
\begin{aligned}
e(t)= & \frac{1}{2}\left\|y_{t}(t)\right\|^{2}+\frac{1}{2}\left(\kappa \square y_{x x}\right)(t)+\frac{1}{2}\left(1-\int_{0}^{t} \kappa(\tau) d \tau\right)\left\|y_{x x}(t)\right\|^{2} \\
& +\frac{1}{2}[\theta(t)-\widetilde{\theta}]^{2} .
\end{aligned}
$$

Then, from (3.3) and (3.4) we obtain

$$
\begin{aligned}
e_{t}(t) & =-\left(g\left(y_{t}(t)\right), y_{t}(t)\right)+\frac{1}{2}\left(\kappa_{t} \square y_{x x}\right)(t)-\frac{1}{2} \kappa(t)\left\|y_{x x}(t)\right\|^{2}-h(t)\left(y_{t}(L, t)\right)^{2} \\
& \leq-\left(g\left(y_{t}(t)\right), y_{t}(t)\right)-\frac{m_{1}}{2}\left(\kappa \square y_{x x}\right)(t)-\frac{1}{2} \kappa(t)\left\|y_{x x}(t)\right\|^{2}
\end{aligned}
$$




$$
-h(t)\left(y_{t}(L, t)\right)^{2} \leq 0, \quad \forall t \geq 0 .
$$

We observe that in view of hypotheses (2.3) and (2.4), we see that $e(t) \geq 0$ and $E(t) \leq \ell^{-1} e(t)$. Therefore it is enough to obtain the desired exponential stability for the modified energy $e(t)$ which will be done below. In order to carry the proof of Theorem 3.2, we need the following Theorem 3.1.

Theorem 3.1. Let $y$ be the solution given by Theorem 2.1 and $e(t)$ be defined by (3.4). Then we have

$$
\begin{aligned}
& \lim _{t \rightarrow \infty} e(t)=0, \quad \lim _{t \rightarrow \infty} h(t) \leq \sqrt{2 r e(0)+(h(0))^{2}}, \\
& h(t) \leq \sqrt{2 r e(0)+(h(0))^{2}}, \quad \forall t \geq 0 .
\end{aligned}
$$

Now, we define the perturbed energy by

$$
e_{\varepsilon}(t)=e(t)+\varepsilon \psi(t), \text { where } \psi(t)=\delta \int_{0}^{t} y(x, t) y_{t}(x, t) d x \text { with } 0<\delta<\frac{1}{2} .
$$

Then, we obtain the following two propositions.

Proposition 3.1. There exists $C_{1}^{\prime}>0$ such that

$$
\left|e_{\varepsilon}(t)-e(t)\right| \leq \varepsilon C_{1}^{\prime} e(t), \quad \forall t \geq 0 \text { and } \forall \varepsilon>0 .
$$

Proof. The proof of this proposition is the same the proof of [15, Proposition $3.1]$, so we will omit this proof.

Proposition 3.2. There exist positive constants $C_{2}^{\prime}, C_{3}^{\prime}$ and $C_{4}^{\prime}$ such that

$$
\begin{gathered}
\frac{d}{d t} e_{\varepsilon}(t) \leq-\varepsilon C_{2}^{\prime} e(t)+\varepsilon C_{3}^{\prime}(1+h(t))(y(L, t))^{2}+\varepsilon C_{4}^{\prime}[\theta(t)-\widetilde{\theta}]^{2}, \\
\forall t \geq 0 \text { and } \forall \varepsilon \in\left[0, \varepsilon_{1}\right] .
\end{gathered}
$$

Proof. Using (1.1), we deduce that

$$
\begin{aligned}
\frac{d}{d t} \psi(t)= & \delta \int_{0}^{L} y_{t}^{2}(t) d x+\delta \int_{0}^{L} y(t) y_{t t}(t) d x \\
= & \delta \int_{0}^{L}\left(y_{t}(t)\right)^{2} d x+\delta \int_{0}^{t} y(t)\left(-y_{x x x x}(t)\right) d x \\
& +\delta \int_{0}^{L} y(t)\left(\int_{0}^{t} \kappa(t-\tau) y_{x x x x}(\tau) d \tau\right) d x \\
& -\delta \int_{0}^{L} y(t) g\left(y_{t}(t)\right) d x .
\end{aligned}
$$

By $y(0, t)=y_{x}(0, t)=y_{x x}(L, t)=0$ and $y \in L^{\infty}(0, T ; W)$, we have the following two inequalities

$$
\int_{0}^{L} y(t)\left(-y_{x x x x}(t)\right) d x
$$




$$
\begin{aligned}
& =-y(L, t) y_{x x x}(L, t)+\int_{0}^{L} y_{x}(t) y_{x x x}(t) d x \\
& =-y(L, t) y_{x x x}(L, t)+y_{x}(L, t) y_{x x}(L, t)-\int_{0}^{L}\left(y_{x x}(t)\right)^{2} d x \\
& =-y(L, t) y_{x x x}(L, t)-\int_{0}^{L}\left(y_{x x}(t)\right)^{2} d x
\end{aligned}
$$

and

$$
\begin{aligned}
& \int_{0}^{L} y(t)\left(\int_{0}^{t} \kappa(t-\tau) y_{x x x x}(\tau) d \tau\right) d x \\
= & y(L, t)\left(\int_{0}^{t} \kappa(t-\tau) y_{x x x}(L, \tau) d \tau\right)-\int_{0}^{L} y_{x}(t)\left(\int_{0}^{t} \kappa(t-\tau) y_{x x x}(\tau) d \tau\right) d x \\
= & y(L, t)\left(\int_{0}^{t} \kappa(t-\tau) y_{x x x}(L, \tau) d \tau\right)-y_{x}(L, t)\left(\int_{0}^{t} \kappa(t-\tau) y_{x x}(L, \tau) d \tau\right) \\
& +\int_{0}^{L} y_{x x}(t)\left(\int_{0}^{t} \kappa(t-\tau) y_{x x}(\tau) d \tau\right) d x \\
= & y(L, t)\left(\int_{0}^{t} \kappa(t-\tau) y_{x x x}(L, \tau) d \tau\right)+\int_{0}^{L} y_{x x}(t)\left(\int_{0}^{t} \kappa(t-\tau) y_{x x}(\tau) d \tau\right) d x .
\end{aligned}
$$

Thus, from (3.8) we get

$$
\begin{aligned}
\frac{d}{d t} \psi(t)= & \delta \int_{0}^{t}\left(y_{t}(t)\right)^{2} d x-\delta y(L, t)\left[y_{x x x}(L, t)-\int_{0}^{t} \kappa(t-\tau) y_{x x x}(L, \tau) d \tau\right] \\
& -\delta \int_{0}^{L}\left(y_{x x}(t)\right)^{2} d x+\delta \int_{0}^{L} y_{x x}(t)\left(\int_{0}^{t} \kappa(t-\tau) y_{x x}(\tau) d \tau\right) d x \\
& -\delta \int_{0}^{L} y(t) g\left(y_{t}(t)\right) d x .
\end{aligned}
$$

From the assumptions on $g$, Young's inequality and Sobolev imbedding theorem, we see that

$$
\delta\left|\int_{0}^{t} y(t) g\left(y_{t}(t)\right) d x\right| \leq M_{1}^{\prime}\left\|y_{t}(t)\right\|^{2}+M_{2}^{\prime}\left\|y_{x x}(t)\right\|^{2},
$$

where $M_{1}^{\prime}$ and $M_{2}^{\prime}$ are some positive constants.

Moreover, for any $\eta>0$, from Fubini's theorem, we have

$$
\begin{aligned}
& \int_{0}^{L} y_{x x}(t)\left(\int_{0}^{t} \kappa(t-\tau) y_{x x}(\tau) d \tau\right) d x \\
= & \int_{0}^{t} \kappa(t-\tau)\left(\int_{0}^{L} y_{x x}(\tau) y_{x x}(t) d x\right) d \tau \\
= & \int_{0}^{t} \kappa(t-\tau)\left(\int_{0}^{L}\left(y_{x x}(\tau)-y_{x x}(t)\right) y_{x x}(t) d x\right) d \tau
\end{aligned}
$$




$$
\begin{aligned}
& +\int_{0}^{t} \kappa(t-\tau)\left(\int_{0}^{L}\left(y_{x x}(t)\right)^{2} d x\right) d \tau \\
\leq & \eta\left\|y_{x x}(t)\right\|^{2}+\frac{1}{4 \eta} \int_{0}^{t} \int_{0}^{L}(\kappa(t-\tau))^{2}\left(y_{x x}(\tau)-y_{x x}(t)\right)^{2} d x d \tau \\
& +\int_{0}^{t} \kappa(\tau)\left(\int_{0}^{L}\left(y_{x x}(t)\right)^{2} d x\right) d \tau \\
\leq & \eta\left\|y_{x x}(t)\right\|^{2}+\frac{1}{4 \eta}\|\kappa\|_{L^{\infty}(0, \infty)}\left(\kappa \square y_{x x}\right)(t)+\int_{0}^{t} \kappa(\tau) d \tau\left\|y_{x x}(t)\right\|^{2} .
\end{aligned}
$$

Combining (3.9)-(3.11), we obtain

(3.12)

$$
\begin{aligned}
\frac{d}{d t} \psi(t) \leq & \delta \int_{0}^{L}\left(y_{t}(t)\right)^{2} d x-\delta y(L, t)\left[y_{x x x}(L, t)-\int_{0}^{t} \kappa(t-\tau) y_{x x x}(L, \tau) d \tau\right] \\
& -\delta \int_{0}^{t}\left(y_{x x}(t)\right)^{2} d x+M_{1}^{\prime}\left\|y_{t}(t)\right\|^{2}+M_{2}^{\prime}\left\|y_{x x}(t)\right\|^{2}+\eta \delta\left\|y_{x x}(t)\right\|^{2} \\
& +\frac{\delta}{4 \eta}\|\kappa\|_{L^{\infty}(0, \infty)}\left(\kappa \square y_{x x}\right)(t)+\delta \int_{0}^{t} \kappa(\tau) d \tau\left\|y_{x x}(t)\right\|^{2} \\
\leq & \left(\delta+M_{1}^{\prime}\right)\left\|y_{t}(t)\right\|^{2}-\delta y(L, t)\left[h(t) y_{t}(L, t)+(\theta(t)-\widetilde{\theta}) \sin t\right] \\
& +\left(M_{2}^{\prime}+\eta \delta+\delta \int_{0}^{t} \kappa(\tau) d \tau-\delta\right)\left\|y_{x x}(t)\right\|^{2} \\
& +\frac{\delta}{4 \eta}\|\kappa\|_{L^{\infty}(0, \infty)}\left(\kappa \square y_{x x}\right)(t) \\
= & -\delta e(t)+\left(M_{1}^{\prime}+\frac{3}{2} \delta\right)\left\|y_{t}(t)\right\|^{2} \\
& +\left(M_{2}^{\prime}+\eta \delta+\frac{\delta}{2} \int_{0}^{t} \kappa(\tau) d \tau-\frac{\delta}{2}\right)\left\|y_{x x}(t)\right\|^{2} \\
& +\left(\frac{\delta}{2}+\frac{\delta}{4 \eta}\|\kappa\|_{L^{\infty}(0, \infty)}\right)\left(\kappa \square y_{x x}\right)(t) \\
& -\delta y(L, t)\left[h(t) y_{t}(L, t)+(\theta(t)-\widetilde{\theta}) \sin t\right]+\frac{\delta}{2}[\theta(t)-\widetilde{\theta}]^{2} .
\end{aligned}
$$

On the other hand, from (2.2), (3.5), (3.6) and (3.12), we get

$$
\text { (3.13) } \begin{aligned}
\frac{d}{d t} e_{\varepsilon}(t)= & \frac{d}{d t} e(t)+\varepsilon \frac{d}{d t} \psi(t) \\
= & -\left(g\left(y_{t}(t)\right), y_{t}(t)\right)-\frac{m_{1}}{2}\left(\kappa \square y_{x x}\right)(t)-\frac{1}{2} \kappa(t)\left\|y_{x x}(t)\right\|^{2} \\
& -h(t)\left(y_{t}(L, t)\right)^{2}-\varepsilon \delta e(t)+\varepsilon\left(M_{1}^{\prime}+\frac{3}{2} \delta\right)\left\|y_{t}(t)\right\|^{2} \\
& +\varepsilon\left(M_{2}^{\prime}+\eta \delta+\frac{\delta}{2} \int_{0}^{t} \kappa(\tau) d \tau-\frac{\delta}{2}\right)\left\|y_{x x}(t)\right\|^{2}
\end{aligned}
$$




$$
\begin{aligned}
& +\varepsilon\left(\frac{\delta}{2}+\frac{\delta}{4 \eta}\|\kappa\|_{L^{\infty}(0, \infty)}\right)\left(\kappa \square y_{x x}\right)(t) \\
& -\varepsilon \delta y(L, t)\left[h(t) y_{t}(L, t)+(\theta(t)-\widetilde{\theta}) \sin t\right]+\frac{\varepsilon \delta}{2}[\theta(t)-\widetilde{\theta}]^{2} \\
\leq & -\varepsilon \delta e(t)-\left(\mu-\varepsilon\left(M_{1}^{\prime}+\frac{3}{2} \delta\right)\right)\left\|y_{t}(t)\right\|^{2} \\
& -\left(\frac{m_{1}}{2}-\varepsilon\left(\frac{\delta}{2}+\frac{\delta}{4 \eta}\|\kappa\|_{L^{\infty}(0, \infty)}\right)\right)\left(\kappa \square y_{x x}\right)(t) \\
& -\left(\frac{1}{2} \kappa(t)-\varepsilon\left(M_{2}^{\prime}+\eta \delta+\frac{\delta}{2} \int_{0}^{t} \kappa(\tau) d \tau-\frac{\delta}{2}\right)\right)\left\|y_{x x}(t)\right\|^{2} \\
& -h(t)\left(1-\frac{\delta \varepsilon}{2}\right)\left(y_{t}(L, t)\right)^{2} \\
& +\frac{\varepsilon \delta}{2}(1+h(t))(y(L, t))^{2}+\varepsilon \delta[\theta(t)-\widetilde{\theta}]^{2} .
\end{aligned}
$$

Now, we define

$$
\varepsilon_{1}=\min \left\{\frac{2 \mu}{2 M_{1}^{\prime}+3 \delta}, \frac{2 m_{1} \eta}{\delta\left(2 \eta+\|\kappa\|_{L^{\infty}(0, \infty)}\right)}, \frac{\kappa(t)}{2 M_{2}^{\prime}+2 \eta \delta+\delta \int_{0}^{t} \kappa(\tau) d \tau-\delta}, \frac{2}{\delta}\right\} .
$$

Considering $\varepsilon \in\left[0, \varepsilon_{1}\right]$ and choosing $\delta>0$ such that $(2 \eta-\ell) \delta+2 M_{2}^{\prime}>\delta$, then from (3.13) and (3.14), we obtain

$$
\frac{d}{d t} e_{\varepsilon}(t) \leq-\varepsilon \delta e(t)+\frac{\varepsilon \delta}{2}(1+h(t))(y(L, t))^{2}+\varepsilon \delta[\theta(t)-\widetilde{\theta}]^{2} .
$$

This completes the proof of Proposition 3.2.

Proof of Theorem 3.1. From Proposition 3.1, we see that

$$
\left(1-\varepsilon C_{1}^{\prime}\right) e(t) \leq e_{\varepsilon}(t) \leq\left(1+\varepsilon C_{1}^{\prime}\right) e(t), \quad \forall t \geq 0 .
$$

From (3.15) and (3.16), we have

$$
\frac{d}{d t} e_{\varepsilon}(t) \leq \frac{-\varepsilon \delta}{1+\varepsilon C_{1}^{\prime}} e_{\varepsilon}(t)+\frac{\varepsilon \delta}{2}(1+h(t))(y(L, t))^{2}+\varepsilon \delta[\theta(t)-\widetilde{\theta}]^{2} .
$$

Let $C_{\varepsilon}=\varepsilon \delta /\left(1+\varepsilon C_{1}^{\prime}\right)$ and apply Gronwall's inequality to (3.17), we get

$$
\begin{aligned}
e_{\varepsilon}(t) \leq & e^{-C_{\varepsilon} t} e_{\varepsilon}(0)+\frac{\varepsilon \delta}{2} \int_{0}^{t} e^{-C_{\varepsilon}(t-\tau)}(1+h(\tau))(y(L, \tau))^{2} d \tau \\
& +\varepsilon \delta \int_{0}^{t} e^{-C_{\varepsilon}(t-\tau)}[\theta(t)-\widetilde{\theta}]^{2} d \tau \\
\leq & e^{-C_{\varepsilon} t} e_{\varepsilon}(0)+\frac{\varepsilon \delta}{2} \sup _{t \geq 0}[1+|h(t)|] \int_{0}^{t} e^{-C_{\varepsilon}(t-\tau)}(y(L, \tau))^{2} d \tau \\
& +\varepsilon \delta \int_{0}^{t} e^{-C_{\varepsilon}(t-\tau)}[\theta(t)-\widetilde{\theta}]^{2} d \tau
\end{aligned}
$$


Referring to the paper [7], we deduce that

$$
\begin{aligned}
& \int_{0}^{t} e^{-C_{\varepsilon}(t-\tau)}(y(L, \tau))^{2} d \tau \\
\leq & \int_{0}^{\frac{t}{2}} e^{-C_{\varepsilon}(t-\tau)}(y(L, \tau))^{2} d \tau+\int_{\frac{t}{2}}^{t} e^{-C_{\varepsilon}(t-\tau)}(y(L, \tau))^{2} d \tau \\
\leq & \max _{0 \leq \tau \leq \frac{t}{2}} e^{-C_{\varepsilon}(t-\tau)} \int_{0}^{\frac{t}{2}}(y(L, \tau))^{2} d \tau+\max _{\frac{t}{2} \leq \tau \leq t} e^{-C_{\varepsilon}(t-\tau)} \int_{\frac{t}{2}}^{t}(y(L, \tau))^{2} d \tau \\
\leq & e^{-\frac{C_{\varepsilon}}{2} t} \int_{0}^{\frac{t}{2}}(y(L, \tau))^{2} d \tau+\int_{\frac{t}{2}}^{\infty}(y(L, \tau))^{2} d \tau .
\end{aligned}
$$

By Theorem 2.1 and $W \hookrightarrow L^{2}\left(0, T ; L^{2}(0, L)\right), y(L, t) \in L^{2}(0, \infty)$, we obtain

$$
\int_{0}^{t} e^{-C_{\varepsilon}(t-\tau)}(y(L, \tau))^{2} d \tau \rightarrow 0 \text { as } t \rightarrow \infty .
$$

Similarly, we see that

$$
\int_{0}^{t} e^{-C_{\varepsilon}(t-\tau)}[\theta(\tau)-\widetilde{\theta}]^{2} d \tau \rightarrow 0 \text { as } t \rightarrow \infty .
$$

From (3.18), (3.19) and (3.20), we deduce

$$
\lim _{t \rightarrow \infty} e_{\varepsilon}(t)=0
$$

Let $\varepsilon_{0}=\min \left\{\varepsilon_{1}, 1 /\left(2 C_{1}^{\prime}\right)\right\}$, where $C_{1}^{\prime}$ is given in Proposition 3.1.

Since $\varepsilon \leq 1 /\left(2 C_{1}^{\prime}\right)\left(\varepsilon \in\left(0, \varepsilon_{0}\right]\right)$ and from $(3.16)$, we get

$$
\frac{1}{2} e(t) \leq e_{\varepsilon}(t) \leq \frac{3}{2} e(t) .
$$

Therefore, from (3.21) and (3.22), we have

$$
\lim _{t \rightarrow \infty} e(t)=0 \text {. }
$$

Now, we consider the Lyapunov functional $U(t)$ for the system (1.3) as follows:

$$
U(t)=e(t)+\frac{(h(t))^{2}}{2 r} .
$$

Then from (3.5), we see that

$$
U_{t}(t) \leq 0
$$

Since $y_{t}(L, \cdot) \in L^{2}(0, \infty)$, we obtain

$$
\sup _{t \geq 0}\left\{e(t)+\frac{(h(t))^{2}}{2 r}\right\} \leq M_{3}^{\prime},
$$

where $M_{3}^{\prime}>0$ is a constant depending on the initial data.

From $U(t)$ is decreasing, we get

$$
e(\infty)+\frac{1}{2 r}(h(\infty))^{2} \leq e(0)+\frac{1}{2 r}(h(0))^{2}
$$


Thus, from (3.23) and (3.25), we have

$$
h(\infty) \leq \sqrt{2 r e(0)+(h(0))^{2}} .
$$

Since $h(t)$ is nondecreasing, we deduce that

$$
h(t) \leq \sqrt{2 r e(0)+(h(0))^{2}} .
$$

Thus, the proof of Theorem 3.1 is completed.

We can now proceed to state our main exponential stability result.

Theorem 3.2. Let $y$ be the solution of Theorem 2.1 and let $e(t)$ be defined by (3.4). Then there exist constants $K>0$ and $\nu>0$ depending on the initial data such that

$$
E(t) \leq K e^{-\nu t}, \quad \forall t \geq 0 .
$$

Proof. From (3.17), we have

$$
\frac{d}{d t} e_{\varepsilon}(t) \leq-C_{\varepsilon} e_{\varepsilon}(t)+\frac{\varepsilon \delta}{2}(1+h(t))(y(L, t))^{2}+\varepsilon \delta[\theta(t)-\widetilde{\theta}]^{2},
$$

where $C_{\varepsilon}=\delta \varepsilon /\left(1+\varepsilon C_{1}^{\prime}\right)$.

Using $(3.26)$ and by integrating over $(0, t)$ in $(3.27)$ we obtain

$$
\begin{aligned}
e_{\varepsilon}(t) \leq & e_{\varepsilon}(0)-C_{\varepsilon} \int_{0}^{t} e_{\varepsilon}(\tau) d \tau+\frac{\varepsilon \delta}{2} \int_{0}^{t}(1+h(\tau))(y(L, \tau))^{2} d \tau \\
& +\varepsilon \delta \int_{0}^{t}[\theta(t)-\widetilde{\theta}]^{2} d \tau \\
\leq & e_{\varepsilon}(0)-C_{\varepsilon} \int_{0}^{t} e_{\varepsilon}(\tau) d \tau+\frac{\varepsilon \delta}{2}\left(1+\|h\|_{L^{\infty}(0, \infty)}\right) \int_{0}^{t}(y(L, \tau))^{2} d \tau \\
& +\varepsilon \delta \int_{0}^{t}[\theta(t)-\widetilde{\theta}]^{2} d \tau .
\end{aligned}
$$

Since $\int_{0}^{\infty}(y(L, \tau))^{2} d \tau$ and $\int_{0}^{\infty}[\theta(t)-\widetilde{\theta}]^{2} d \tau$ are bounded, by Gronwall's inequality, we deduce that

$$
e_{\varepsilon}(t) \leq\left(K_{1}+e_{\varepsilon}(0)\right) \exp \left(-C_{\varepsilon} t\right)
$$

where $K_{1}$ is some positive constant.

For sufficiently small $\varepsilon\left(0<\varepsilon<\frac{1}{2 C_{1}^{\prime}}\right)$, using Proposition 3.1, we get

$$
E(t) \leq \ell^{-1} e(t) \leq K e^{-\nu t},
$$

where $K=2 \ell^{-1}\left(K_{1}+e_{\varepsilon}(0)\right)$ and $\nu=C_{\varepsilon}$.

The proof of Theorem 3.2 is completed. 


\section{References}

[1] D. Andrade and J. M. Rivera, Exponential decay of non-linear wave equation with a viscoelastic boundary condition, Math. Methods Appl. Sci. 23 (2000), no. 1, 4161.

[2] J. M. Ball, Initial-boundary value problems for an extensible beam, J. Math. Anal. Appl. 42 (1973), 61-90.

[3] F. Conrad and O. Omër, On the stabilization of a flexible beam with a tip mass, SIAM J. Control Optim. 36 (1998), no. 6, 1962-1986.

[4] M. Dahleh and W. Hopkins, Adaptive stabilization of single-input single-output delay systems, IEEE Trans. Automat. Control 31 (1986), no. 6, 577-579.

[5] M. Feckan, Free vibrations of beams on bearings with nonlinear elastic responses, J. Differential Equations 154 (1999), no. 1, 5572.

[6] E. Feireisl, Nonzero time periodic solutions to an equation of Petrovsky type with nonlinear boundary condition: slow oscillations of beams on elastic bearings, Ann. Scuola Norm. Sup. Pisa Cl. Sci. (4) 20 (1993), no. 1, 133-146.

[7] B. Z. Guo and W. Guo, Adaptive stabilization for a Kirchhoff-type nonlinear beam under boundary output feedback control, Nonlinear Anal. 66 (2007), no. 2, 427-441.

[8] B. Z. Guo and Z. H. Luo, Initial-boundary value problem and exponential decay for a flexible-beam vibration with gain adaptive direct strain feedback control, Nonlinear Anal. 27 (1996), no. 3, 353-365.

[9] M. Kirane and N. E. Tatar, A memory type boundary stabilization of a mildly damped wave equation, Electron. J. Qual. Theory Differ. Equ. 1999 (1999), no. 6, 1-7.

[10] I. Lasiecka, Exponential decay rates for the solutions of Euler-Bernoulli equations with boundary dissipation occurring in the moments only, J. Differential Equations 95 (1992), no. 1, 169-182.

[11] J. L. Lions and E. Magenes, Non-homogeneous Boundary Value Problems and Applications, Springer-Verlag, New York, 1972.

[12] H. Ma, Uniform decay rates for the solutions to the Euler-Bernoulli plate equation with boundary feedback via bending moments, Differential Integral Equations 5 (1992), 1121-1150.

[13] T. F. Ma, Boundary stabilization for a non-linear beam on elastic bearings, Math. Methods Appl. Sci. 24 (2001), 583-594.

[14] J. Y. Park, Y. H. Kang, and J. A. Kim, Existence and exponential stability for a EulerBernoulli beam equation with memory and boundary output feedback control term, Acta Appl. Math. 104 (2008), no. 3, 287-301.

[15] J. Y. Park and J. A. Kim, Existence and uniform decay for Euler-Bernoulli beam equation with memory term, Math. Methods Appl. Sci. 27 (2004), no. 14, 1629-1640.

[16] S. K. Patcheu, On a global solution and asymptotic behaviour for the generalized damped extensible beam equation, J. Differential Equations 135 (1997), no. 2, 299314.

[17] M. L. Santos, Asymptotic behaviour of solutions to wave equations with a memory condition at the boundary, Electron. J. Differential Equations 2001 (2001), no. 73, 111.

YONG HAN KANG

INSTITUTE OF LiBERAL EDUCATION

Catholic University of Daegu

GyeOngSAN 712-702, KOREA

E-mail address: yonghann@cu.ac.kr 
JoNG YEOUL PARK

Department of Mathematics College of Science

Pusan National University

Pusan 609-735, Korea

E-mail address: jyepark@pusan.ac.kr

Jung Ae Kim

Department of Mathematics College of Science

Hanbat National University

DAEJEON 305-719, KorEA

E-mail address: jakim@nims.re.kr 